\title{
Nonlinear Evolutionary Mechanisms of Instability of Plane-Shear Slope: Catastrophe, Bifurcation, Chaos and Physical Prediction
}

\author{
By \\ S. Q. Qin ${ }^{1}$, J. J. Jiao ${ }^{2}$, and Z. G. Li ${ }^{1}$ \\ ${ }^{1}$ Engineering Geology and Applied Geophysics Department, Institute of Geology \\ and Geophysics, Chinese Academy of Sciences, Beijing, P.R. China \\ ${ }^{2}$ Department of Earth Sciences, The University of Hong Kong, Hong Kong, P.R. China
}

Received July 13, 2004; accepted January 12, 2005

Published online March 31, 2005 (C) Springer-Verlag 2005

\begin{abstract}
Summary
A cusp catastrophe model is presented and the necessary and sufficient conditions leading to landslides are discussed. The sliding surface is assumed to be planar and is a combination of two media: medium 1 is elastic-brittle or strain-hardening and medium 2 is strain-softening. The shear stress-strain constitutive model for the strain-softening medium is described by the Weibull's distribution law. This paper is a generalization and extension of the paper by Qin et al. (2001b), in which the shear stress-strain constitutive model for medium 2 was described by a negative exponent distribution; a special case of the Weibull's distribution law. It is found that the instability of the slope relies mainly on both the stiffness ratio of the media and the homogeneity index and that a new role of water is to enlarge the material homogeneity or brittleness and hence to reduce the stiffness ratio. A nonlinear dynamic model (also called a physical forecasting model), which is derived by considering the time-dependent behavior of the strain-softening medium, is used to study the time prediction of landslides. An algorithm of inversion on the nonlinear dynamic model is suggested for seeking the precursory abnormality and abstracting mechanical parameters from the observed series of a landslide. A case study of the Jimingsi landslide is analysed and its nonlinear dynamic model is established from the observation series of this landslide using the suggested model and the algorithm of inversion. It is found that the catastrophic characteristic index $|D|$ shows a quick rise till reaching an extremely high peak value after the slope evolves into the tertiary creep, and subsequently approaches a zero value prior to instability, which can be regarded as an important precursory abnormality index. By taking into account the evolutionary characteristic of the slope being in the secondary creep, a simplified nonlinear dynamic model is proposed for studying the properties of bifurcation and chaos. It is shown that the emergence of chaos depends on the mechanical parameters of the sliding-surface media.
\end{abstract}

Keywords: Cusp catastrophe, instability, stiffness ratio, homogeneity index, chaos, physical prediction. 


\section{Introduction}

The commonly used limit equilibrium approach for the stability analysis of slopes, which is incapable of considering the creep displacement along the sliding surface, may result in an unconvincing assessment of slope stability. Many numerical methods, such as finite-element, boundary-element, and discrete-element methods, can do well in simulating the nonlinear behavior of slope deformation and failure, but most of them are not suitable for modeling the nonlinear behavior of brittle rock (Tang, 1997). However, there is a tendency in this field that the theoretical framework for nonlinear and discontinuum problems becomes more and more complicated and sometimes becomes less practicable. Hence, it is suggested that a convenient and practical approach to evaluate the stability of a slope should be developed which allows for the displacement along the sliding surface.

The common methods for predicting the failure time of landslides are empirical and statistical. For example, Saito (1969) forecasted the time of slope failure by tertiary creep. Voight (1989) presented a multi-parameter-forecasting formula according to the statistical function among time, displacement and empirical constants. Qin and Wang (2000) suggested a homomorphic model for identifying abrupt abnormalities of landslide forerunners. These statistical predictions, as pointed out by Rat (1988), are generally very unreliable and often very tricky due to lack of physical basis. The essential route for landslide prediction is to turn an empirical or statistical prediction into a physical one.

The methods and approaches based on nonlinear dynamic systems (NDS) theory have been recently applied to investigate various problems in rock mechanics and geomechanics, including landslides (Saunders, 1980; Keilis-Borok, 1990; Qin et al., 1993; Phillips, 1995; and Qin et al., 2001). A brief review of these applications can be found in the papers by Qin et al., 2001. Qin et al. (2001a) developed a cusp catastrophe model of instability of slip-buckling slope, and hence analyzed the mechanisms of instability as well as the connection between the slope state and behavior in creep. Qin et al. (2001b) also studied the unstable mechanisms of a plane-shear slope and the chaotic dynamic mechanisms of its evolutionary process by NDS theory. They assumed that the sliding surface of the landslides was planar and was a combination of two media: medium 1 was elastic-brittle and medium 2 was strain-softening. The shear stress-strain constitutive model for medium 2 was described by a negative exponent distribution.

This paper is a generalization and extension of the paper by Qin et al. (2001b). The sliding surface of the landslide is also assumed to be planar and is a combination of two media, but the medium 1 can be elastic-brittle or strain-hardening. The shear stress-strain constitutive model for the strain-softening medium is described by the Weibull's distribution law, which is more general and practical than a negative exponent distribution. The discriminating criteria leading to a fast-moving landslide will be suggested. A nonlinear dynamic model derived by considering the time-dependent behavior of the strain-softening medium will be used to study the time prediction of landslides. An algorithm of inversion on the nonlinear dynamic model will be suggested for seeking the precursory abnormality and abstracting mechanical parameters from the observed series of a landslide. A case study of the Jimingsi landslide will be used to demonstrate the applicability of the model. 


\section{Cusp Catastrophe Model of a Plane-Shear Slope and its Instability Mechanisms}

\subsection{Mechanical Model}

Figure 1 shows the model used in this study. The sliding surface with the dip angle $\theta$ is a nonuniform intercalation, the rock mass with the weight $W g$ (g is gravity acceleration) above is a rigid body. Under the action of the driving force caused by the weight of the rock mass, the creep displacement is $u$ along the intercalation. Due to different stress levels, material composition, textures and structures at different segments of the intercalation, the interaction can comprise many kinds of media with different mechanical properties, such as elastic-brittle, elastic-ductile (strain-hardening) and strain-softening, etc. To simplify the analysis, we regard the interaction media as a combination of only two kinds of media with different mechanical properties, i.e., one (medium 1) possesses a complicated elastic-brittle (such as a strong interlocking rock block or so-called rock bridge) or strain-hardening (such as hard clay or incompact sandy soil) behavior and the other (medium 2) has a strain-softening property. Whether medium 1 is elastic-brittle or is strain-hardening, the same conclusions will be drawn in this paper. For simplicity, we will consider the elastic-brittle property of medium 1 in the analysis below.

The shear stress-displacement relation, for the strong rock block with pre-existing multi-cracks or flaws, can generally be assumed to be linear prior to failure. At failure, the rock block is bisected and a sudden stress drop will take place. After failure of the rock block the combined cracks could resemble a stepped or sawtoothed, throughgoing discontinuity, resulting in a slow, sometimes quick (e.g. Lajitai, 1967, and Gehle and Kutter, 2003) stress increment instead of a decrease with growing displacement. Finally, the stepped or sawtoothed discontinuity is levelled out, which may lead to a rapid stress decrease with growing displacement. According to the above assumptions and direct shear tests on intermittent rock joints performed by Gehle and Kutter (2003), the constitutive model for medium 1 (Fig. 2) can be written as

$$
\tau=\left\{\begin{array}{l}
G_{e 1} \frac{u}{h}\left(u<u_{b}\right) \\
G_{e 2} \frac{u}{h}\left(u_{b} \leq u \leq u_{2}\right),
\end{array}\right.
$$

where $u_{b}$ is the critical displacement corresponding to the failure of the rock block, $u_{2}$ is the displacement when the stepped or sawtoothed discontinuity inside the rock

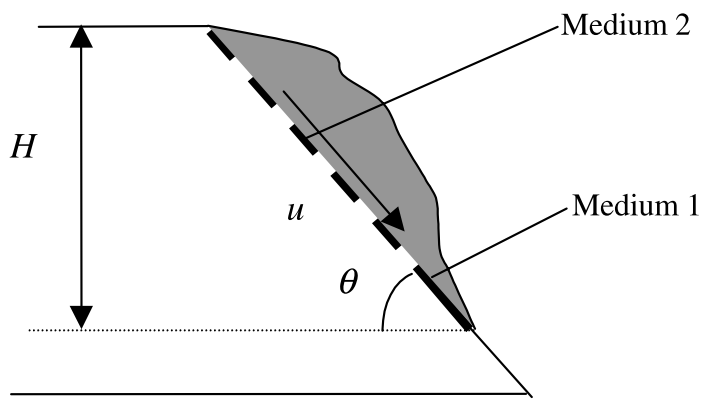

Fig. 1. Mechanical model of a plane-shear slope instability 


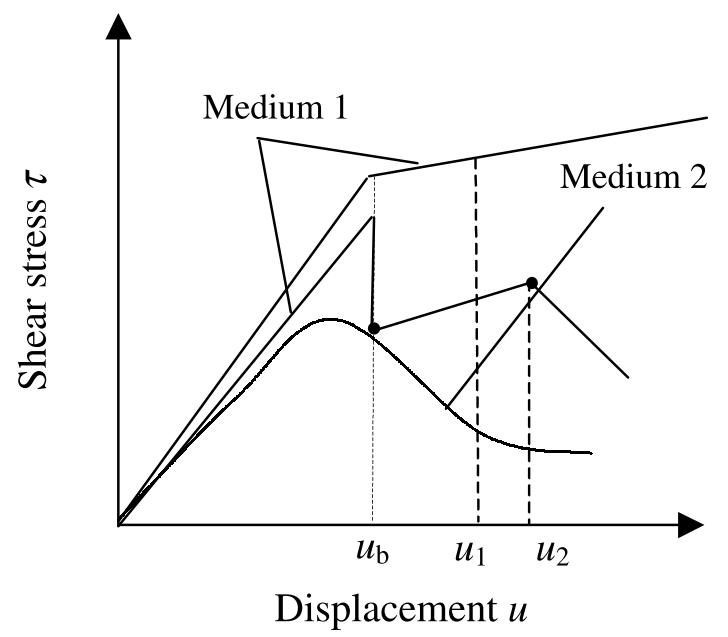

Fig. 2. Constitutive curves of two media along the sliding plane

block is levelled out, $G_{e 1}$ and $G_{e 2}$ are the shear moduli for $u<u_{b}$ and $u_{b} \leq u \leq u_{2}$, respectively, and $h$ is the layer thickness of the intercalation.

A simplified constitutive model of medium 2 can generally be expressed as a nonlinear function between the shear stress $\tau$ and the creep displacement $u$. Qin et al. (2001b) used a negative exponent distribution of strength to describe the strain-softening property. However, the most commonly used distribution is the Weibull's distribution which describes very well the experimental data (Hudson and Fairhurst, 1969), so the Weibull's distribution law is adopted for the strain-softening media, i.e.

$$
\tau=G_{s} \frac{u}{h} \exp \left[-\left(\frac{u}{u_{0}}\right)^{m}\right],
$$

where $G_{s}$ is the initial shear modulus, $u_{0}$ is a measurement of average strength and $m$ is the shape parameter (Fig. 3). One of the attractive aspects of the Weibull's distribution

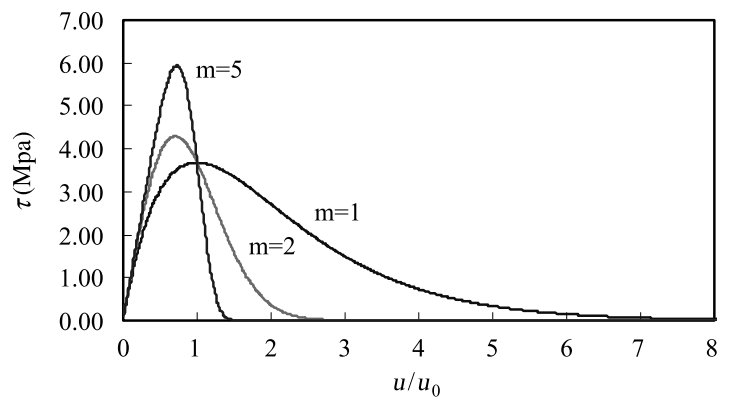

Fig. 3. Weibull's distribution constitutive curves of medium 2 for different values of $m\left(G_{\mathrm{s}}=10 \mathrm{MPa}\right.$, $u_{0}=0.1 \mathrm{~m}$, and $\left.h=0.1 \mathrm{~m}\right)$ 
is the presence of the shape parameter which allows this function to take a wide variety of shapes (Fig. 3). For instance, for $m=1$, this distribution is exponential; at about $m=2$, it very closely approximates a normal distribution. Since $m$ is a measurement of the local strength variability, it can be considered as a homogeneity index (Tang, 1993). The larger the index $m$, the more homogeneous is the rock. When $m$ trends to infinity, the variance trends to zero and a "perfect" rock is obtained. A material with such a property is so-called ideal brittle material, such as glass. Thus, the shape parameter $m$ can also be referred to as the brittleness index.

\subsection{Cusp Catastrophe Model}

The overall potential energy for the system illustrated in Fig. 1 is equal to the sum of the strain energy and driving potential energy, i.e.

$$
V=\left\{\begin{array}{c}
l_{s} \int_{0}^{u} \frac{G_{s} u}{h} \exp \left[-\left(\frac{u}{u_{0}}\right)^{m}\right] d u+\frac{1}{2} \frac{G_{e 1} l_{e}}{h} u^{2}-W g u \sin \theta\left(u<u_{b}\right) \\
l_{s} \int_{0}^{u} \frac{G_{s} u}{h} \exp \left[-\left(\frac{u}{u_{0}}\right)^{m}\right] d u+\frac{1}{2} \frac{G_{e 2} l_{e}}{h} u^{2}+\frac{1}{2} \frac{l_{e} u_{b}^{2}}{h}\left(G_{e 1}-G_{e 2}\right) \\
-W g u \sin \theta\left(u_{b} \leq u \leq u_{2}\right),
\end{array}\right.
$$

where $l_{e}$ and $l_{s}$ are the length of the sliding surface for media 1 and 2, respectively, and $l_{s}+l_{e}=H / \sin \beta ; u$ can be regarded as the state variable in the cusp catastrophe analysis. It is assumed here that $l_{e}$ is far less than $l_{s}$, because a slope probably is very stable when $l_{e}>l_{s}$.

Let $V^{\prime}=0$ and the equilibrium surface equation (Fig. 4) is expressed as

$$
V^{\prime}=\frac{G_{s} l_{s}}{h} u \exp \left[-\left(\frac{u}{u_{0}}\right)^{m}\right]+\frac{G_{e} l_{e}}{h} u-W g \sin \theta=0,
$$

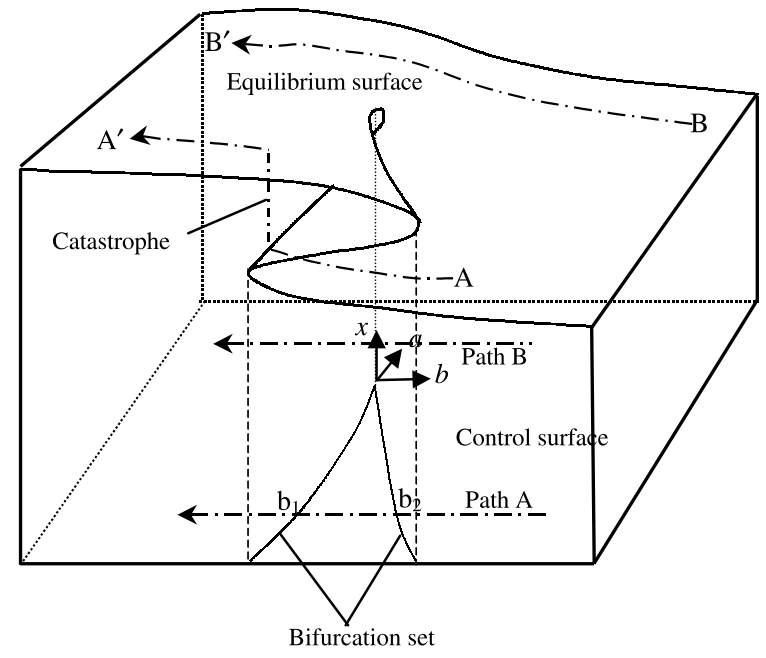

Fig. 4. Cusp catastrophe model 
where $G_{e}=G_{e 1}\left(u<u_{b}\right)$ or $G_{e}=G_{e 2}\left(u_{b} \leq u \leq u_{2}\right)$. Equation (4) is the equilibrium condition of forces. By the smoothness property of the equilibrium surface, the cusp can be solved using the condition $V^{\prime \prime \prime}=0$, i.e.

$$
u=u_{1}=\left(\frac{m+1}{m}\right)^{\frac{1}{m}} u_{0}
$$

Equation (5) shows that the displacement value at cusp is exactly the displacement value at the turning point of the constitutive curve of medium 2.

By Taylor series expansion with respect to $u_{1}$ for Eq. (4), discarding all the terms but the first three because the third order term is the minimum one away from zero, and substituting Eq. (5) into Eq. (4), one has:

$\frac{1}{6} \frac{G_{s} l_{s} u_{1} \exp \left(-\frac{m+1}{m}\right) m(m+1)^{2}}{h}\left[\begin{array}{l}\left(\frac{u-u_{1}}{u_{1}}\right)^{3}+6\left(\frac{G_{e} l_{e} \exp \left(\frac{m+1}{m}\right)}{G_{s} l_{s} m(m+1)^{2}}-\frac{1}{(m+1)^{2}}\right)\left(\frac{u-u_{1}}{u_{1}}\right) \\ +\frac{6}{m(m+1)^{2}}\left(1+\frac{G_{e} l_{e} \exp \left(\frac{m+1}{m}\right)}{G_{s} l_{s}}-\frac{W g h \sin \theta \exp \left(\frac{m+1}{m}\right)}{G_{s} l_{s} u_{1}}\right)\end{array}\right]=0$

In order to transform Eq. (6) into a standard form of cusp catastrophe, let

$$
\begin{gathered}
x=\frac{u-u_{1}}{u_{1}}, \\
a=\frac{6}{(m+1)^{2}}(k-1), \\
b=\frac{6}{m(m+1)^{2}}(1+m k-\xi), \\
k=\frac{G_{e} l_{e} \exp \left(\frac{m+1}{m}\right)}{G_{s} l_{s} m}, \\
\xi=\frac{W g h \sin \theta \exp \left(\frac{m+1}{m}\right)}{G_{s} l_{s} u_{1}},
\end{gathered}
$$

where $k$ is the ratio of the stiffness of medium $1\left(k_{e}=G_{e} l_{e} / h\right)$ to the stiffness at the turning point of the constitutive curve of medium $2\left(k_{s}=\mid-G_{s} l_{s} m \exp [-(m+\right.$ $1 / m)] / h \mid$ ) (for simplicity, it is called hereafter the stiffness ratio); $\xi$ is relative to the weight of rock mass, geometric size of the system, and mechanical parameters of media (referred to as the geometric-mechanical parameter).

Substituting Eqs. (7)-(11) into Eq. (6) leads to

$$
x^{3}+a x+b=0 \text {. }
$$

Equation (12) is the standard cusp catastrophe model of the equilibrium surface, with $a$ and $b$ as its control parameters and $x$ as its state variable.

The cusp catastrophe described by the equilibrium surface containing fold or pleat is illustrated in Fig. 4 where axes of three-dimensional space are the control parameters $a, b$ (horizontal) and response parameter $x$ (vertical). As pointed out by Henley 
(1976), path $\mathrm{B}-\mathrm{B}^{\prime}$ and path $\mathrm{A}-\mathrm{A}^{\prime}$ denote a stable evolutionary process and an unstable evolutionary process, respectively. The bifurcation set (cusp) (Thom, 1972) can be expressed as

$$
D=4 a^{3}+27 b^{2}=0
$$

Substituting Eqs. (8) and (9) into Eq. (13) leads to

$$
D=4 \beta^{3}(k-1)^{3}+27\left(\frac{\beta}{m}\right)^{2}(1+m k-\xi)^{2}=0
$$

where $\beta=6 /(m+1)^{2}$.

The bifurcation set (Fig. 4) defines the thresholds where sudden changes can take place. As long as the state of the system remains outside the bifurcation set $(D>0)$, the behavior varies smoothly and continuously as a function of the control parameters. Even on entering the bifurcation set $(D<0)$ no abrupt change is observed. When the control point passes all the way through the bifurcation set $(D=0)$, however, a catastrophe is inevitable. Thus, Eqs. (14) or (13) is the sufficient and necessary mechanical criteria for the plane-shear slope instability (fast-moving landslide). In the following analysis $D$ is referred to as the catastrophic characteristic index.

Obviously, only when $k \leq 1$, the condition of Eq. (14) may be satisfied. Thus, the necessary condition of instability is

$$
k=\frac{G_{e} l_{e} \exp \left(\frac{m+1}{m}\right)}{G_{s} l_{s} m} \leq 1
$$

Equation (15) shows that the smaller the stiffness of medium 1 is, the larger the post-peak stiffness (the absolute value of post-peak stress constitutive curve slope) of medium 2, and the more possible it is for the slope system to lead to catastrophe. It is known from Eq. (15) that $k$ decreases with an increase of $m$ for the fixed values of $G_{\mathrm{e}} l_{\mathrm{e}} / G_{\mathrm{s}} l_{\mathrm{s}}$, demonstrating more prone to landsliding for a more homogenous rock.

It is generally believed that the instability of a slope has a close connection with the action of water, which reduces the anti-shear strength and increases the pore-water or uplift pressure in the intercalation. Figure 5 shows that water also has a new role of enlarging the homogeneity or brittleness index $m$ and hence reducing the stiffness ratio, more readily leading to landsliding.

\subsection{Symbol $b$ and the Regions with Creep Behaviour}

Substituting Eqs. (10) and (11) into Eq. (9) leads to

$$
b=\frac{6 \exp \left(\frac{m+1}{m}\right)}{m(m+1)^{2} G_{s} l_{s} u_{1}}\left[G_{e} l_{e} u_{1}+G_{s} l_{s} u_{1} \exp \left(-\frac{m+1}{m}\right)-W g h \sin \theta\right],
$$

$b$ depends on the relative magnitude of resisting and driving forces at the turning point of the strain-stress curve of medium 2. It is clear from Eq. (16) that $b>0, b=0$ and $b<0$ correspond to the sliding acceleration of the slope mass: $<0$ (primary creep), $=0$ (secondary creep) and $>0$ (tertiary creep), respectively. Figure 6 illustrates the corresponding relation between $b$ and the regions with creep behaviour. 


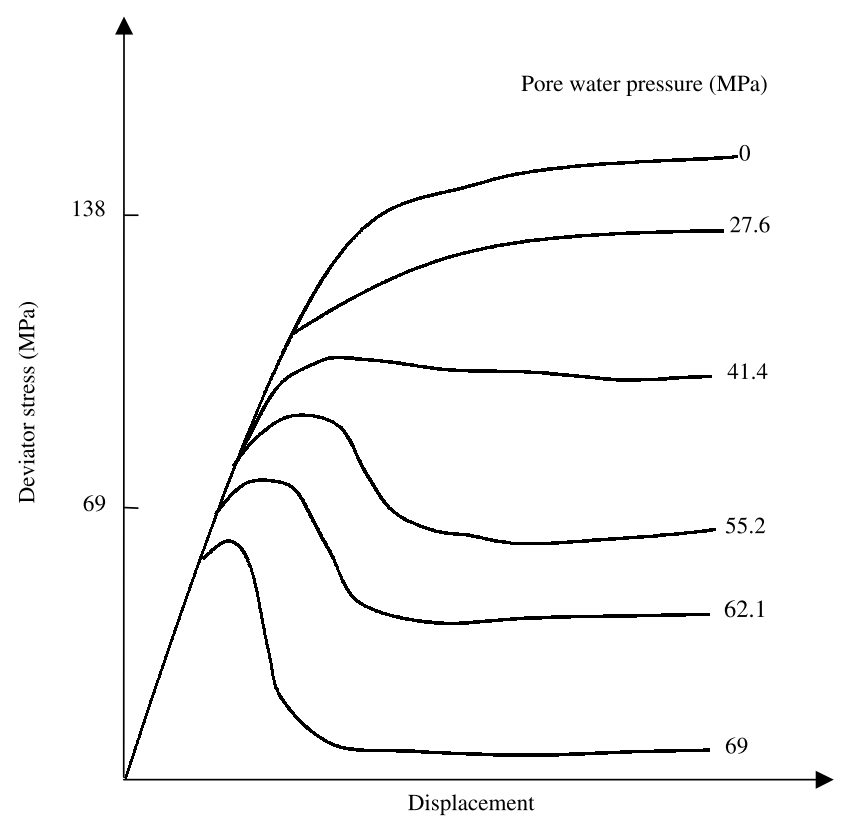

Fig. 5. The effect of pore-pressure on the brittle-ductile transition in limestone at a confining pressure of 6.9 MPa. Numbers on the curves are values of pore pressure in MPa (Fig. 8.9.2 in Jaeger and Cook, 1979)

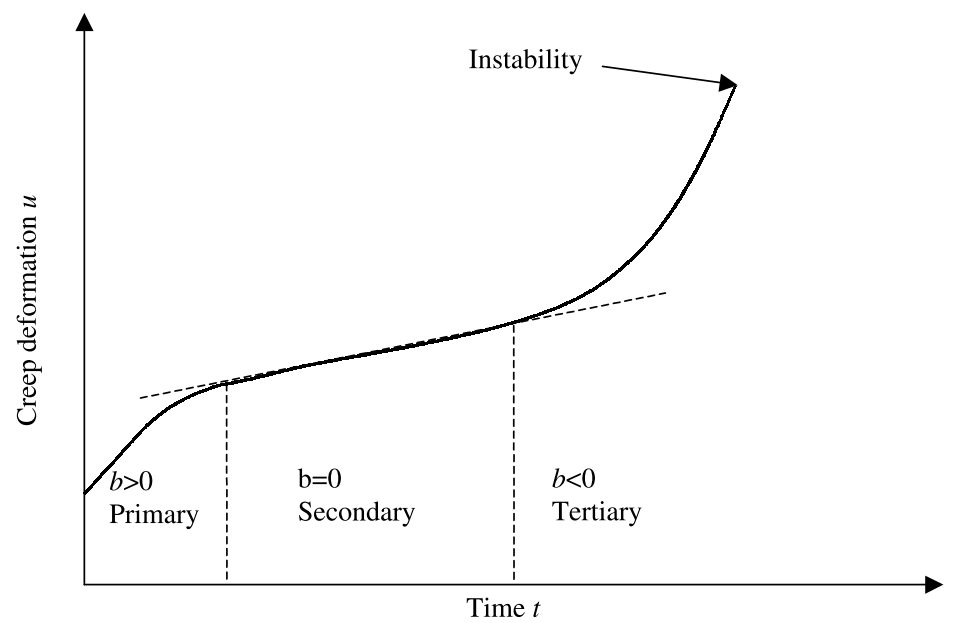

Fig. 6. Regions of behavior in creep. Primary, secondary and tertiary creep correspond to decreasing rate, steady rate and increasing rate with time, respectively

Equation $(12)(b<0)$ can be used to determine the critical displacement value at the catastrophic points corresponding to failure of medium 1 as follows:

$$
u_{b}=u_{1}\left[1-\frac{\sqrt{2}}{m+1}(1-k)^{1 / 2}\right](k \leq 1)
$$




\section{Nonlinear Dynamic Model of Evolutionary Process of Slope}

In the above analysis, we considered the quasi-static motion of the slope, but not the dynamic process of instability. To study the chaotic mechanism of the evolutionary process of a slope and to predict the landslide instability, a nonlinear dynamic model is needed.

\subsection{Nonlinear Dynamic Model}

If the viscosity or creep (i.e. time-dependent) property of the strain-softening medium is considered (Fig. 7), then its shear strength can be expressed as

$$
\tau=\tau_{s}+\tau_{v}=G_{s} \frac{u}{h} \exp \left[-\left(\frac{u}{u_{0}}\right)^{m}\right]+\frac{\eta}{h} \frac{d u}{d t}
$$

where $\eta$ is the viscosity coefficient. Substituting Eq. (18) into the equilibrium surface Eq. (4) and using Eqs. (5)-(11), one obtains

$$
\frac{d x}{d t}=-\frac{1}{6} \frac{G_{s} \exp \left(-\frac{m+1}{m}\right) m(m+1)^{2}}{\eta}\left(x^{3}+a x+b\right) .
$$

Equation (19) is a nonlinear dynamic or a physical forecasting model with a definite physical meaning of each parameter. As long as we know the parameters of the model by means of laboratory tests and field investigations, the prediction of the landslide behavior can be made. It is known from the above-mentioned analysis that the parameters $a$ and $b$ denote the possible instability and the creep phase of a landslide, respectively. Obviously, the dimensionless displacement rate grows as the parameter $b(b<0)$ decreases when $a<0$.

Now, we study the character of the equilibrium state as given by Eq. (19). It is known by letting $\mathrm{d} x / \mathrm{d} t=0$ that Eq. (19) also is a cusp catastrophe and its instability condition is the same as Eq. (13). It is realized from Eq. (19) that the dimensionless displacement $x$ is wholly dominated by the mechanical and geometric parameter of the slope body itself. Thus, it is deduced that the variations of the mechanical parameters can be reflected in the observed displacement-time series of a landslide when the geometric parameters are fixed, which demonstrates that we can estimate the mechanical parameters from the observation series using an algorithm of inversion.

\subsection{Algorithm of Inversion on the Nonlinear Dynamic Model}

The only information available at present is the data derived from observation and description of deformation and failure phenomena for the complex evolution process

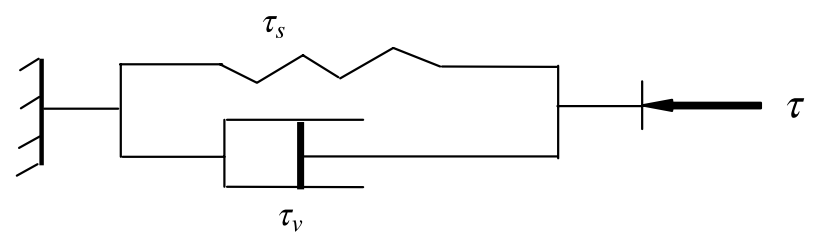

Fig. 7. A shear stress model considering the viscosity or creep property, similar to Kelvin or Voigt model 
of landslides. That is to say, we know a series of specific solutions of the dynamic model. If we regard such solutions as a series of discrete values of the dynamic model, the quasi-ideal nonlinear dynamic model for the evolution of the landslide can, thus, be obtained through an algorithm of inversion. The following gives the analytical procedures and steps.

(1) Substituting Eqs. (7)-(11) into Eq. (19) in order to transforming $x$ - $t$ series into $u$ - $t$ series, one has

$$
\frac{d u}{d t}=c_{1} u^{3}+c_{2} u^{2}+c_{3} u+c_{4}
$$

where

$$
\begin{gathered}
c=-G_{s} \exp \left(-\frac{m+1}{m}\right) m(m+1)^{2} / 6 \eta, \\
c_{1}=c / u_{1}^{2}, \\
c_{2}=-3 c / u_{1}, \\
c_{3}=(3+a) c \\
c_{4}=[b-(a+1)] c u_{1} .
\end{gathered}
$$

(2) The parameters $c_{1}, c_{2}, c_{3}$ and $c_{4}$ can be solved by best fitting the original observation data with Eq. (20) by a fitting algorithm. It should be noted (Bakus and Gilbert, 1970) that, in most cases, the solutions of Eq. (20) usually are unstable when the least squares method is adopted. Therefore, it is suggested that an improved iterative algorithm of inversion presented by Qin et al. (2002) should be applied to the solution of Eq. (20). The predicted values can be calculated using the Runge-Kutta integration method.

\subsection{Case Study}

The Jimingsi landslide at Zigui county in Hubei province, PR China, with a volume of ca. $6 \times 10^{5} \mathrm{~m}^{3}$, occurred on 29 June 1991 (Fig. 8). The landslide mass consists of hard limestone containing soft and thin marlite intercalations. The landslide was induced by mining limestone and is a typical plane-shear instability with a slope angle of $35^{\circ}$. The upper elevation of the stored ore at the site is $250 \mathrm{~m}$. The limestone below elevation $300-310 \mathrm{~m}$ was exploited, forming a steep cutting surface with a height of 50-70 m and width of $153 \mathrm{~m}$. Fractures at the two sides of the landslide mass were present and formed the side boundaries. The upper part of the landslide mass was cut off by a fault. This slope with a soft marlite sliding surface was characterised by a set of boundary conditions leading to landslide instability. Under such conditions, due to beast vibrations and rainfalls, the slope body began to deform. The annual average rainfall in this area is $1200 \mathrm{~mm}$. The total rainfall was $106.8 \mathrm{~mm}$ from 1 May to 15 June in 1991. 


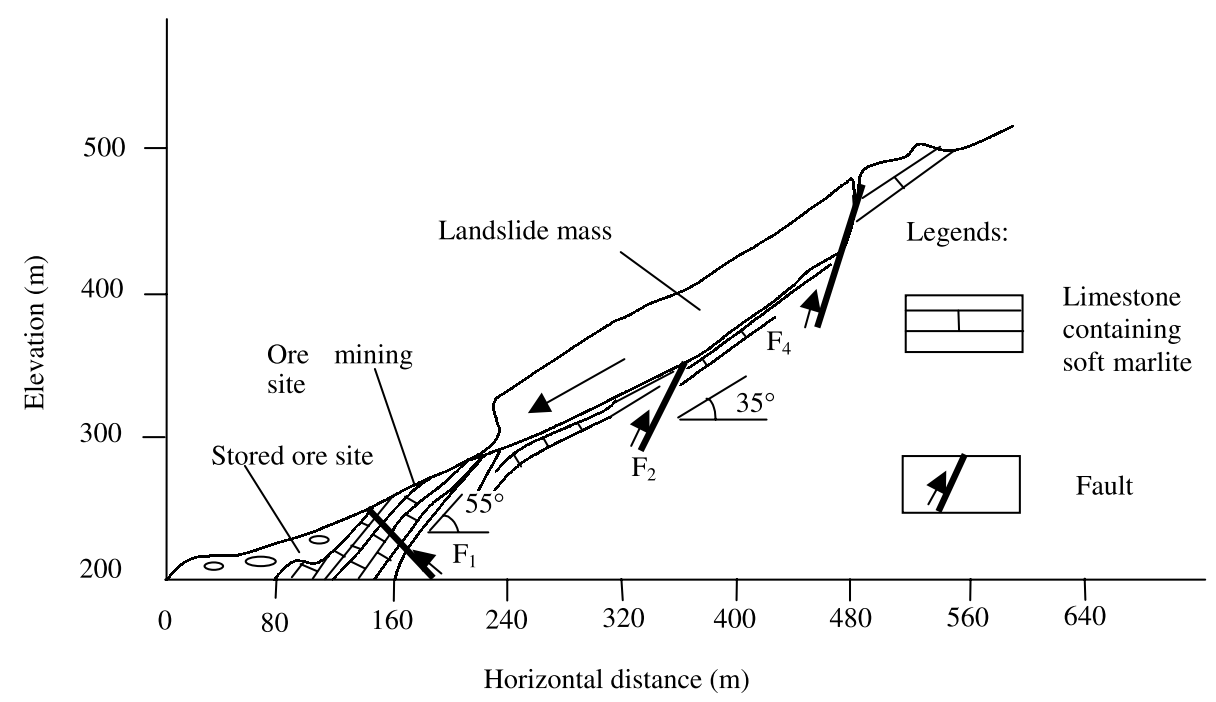

Fig. 8. Geological section of Jimingsi landslide

On 5 March 1990, a few cracks at the top of the landslide were observed. Since 24 April 1990, an increase in the crack width was measured with an automatic monitoring system. The monitored crack width at C4 and C7 observation points (Lu, 1994) are illustrated in Fig. 9. The displacement (crack width) curves versus time are very similar to the typical creep curves shown in Fig. 6.

At $\mathrm{C} 4$ observation point, the nonlinear dynamic model obtained using the abovementioned algorithm of inversion is as follows:

$$
\frac{d x}{d t}=-0.5161\left(x^{3}-1.03701 x-0.4282\right) .
$$

Figure 9 shows that the predicted values are in good agreement with the observed values. By choosing the data between the beginning of the observation and a certain time, we can get a series of values for $a, b$ and $D$. It is also observed from Fig. 9 that the catastrophic characteristic index $|D|$ behaves as a relatively steady change during the secondary creep phase, then, it begins to have a rapid increase and reaches an extremely high peak value after entering into the tertiary creep, and eventually it quickly falls to approximately zero prior to the landslide instability as expected. Thus, the index $D$ can be regarded as an important index indicating the precursory abnormality of the landslide.

Taking the observation series at $\mathrm{C} 4$ observation point as an example, we can estimate the possible parameter scales using the conditions $a=-1.03701$ and $b=-0.4282$ obtained from Eq. (26). It is seen from Fig. 10 that the parameters $k$, $\xi$ and $m$ are in the range $0 \leq k \leq 0.8272,1 \leq \xi \leq 1.61$ and $0<m \leq 1.4$, respectively.

Considering the worst scenario, i.e., $l_{\mathrm{e}}=0$ (then, $k=0, m=1.4$ and $\xi=1.582$ ), and applying the known conditions of $l_{\mathrm{s}}=237.6 \mathrm{~m}, u_{1}=0.928 \mathrm{~m}, W \mathrm{~g}=641520 \mathrm{kN}$, $h=0.2 \mathrm{~m}$ and $\theta=35^{\circ}$, we obtain $G_{s}=1.17 \mathrm{MPa}$ and $\eta=7.128 \times 10^{10} \mathrm{~Pa}$.s, and realize that the shear modulus is very low, close to landslide instability. 


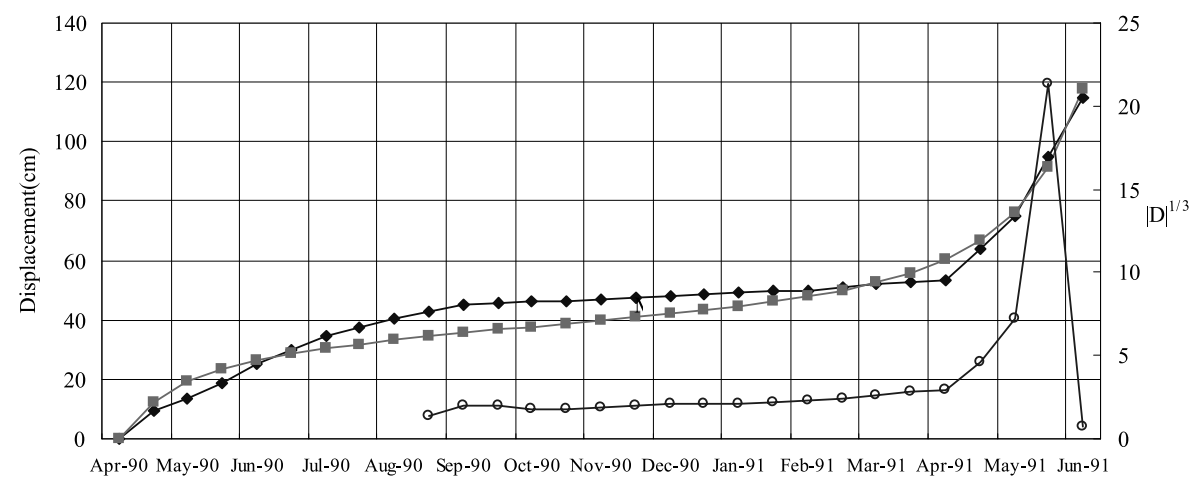

Time(months)

(a)

$$
\multimap-\text { Monitoring series } \longrightarrow \text { Forecasting series } \multimap-\mathrm{D}
$$

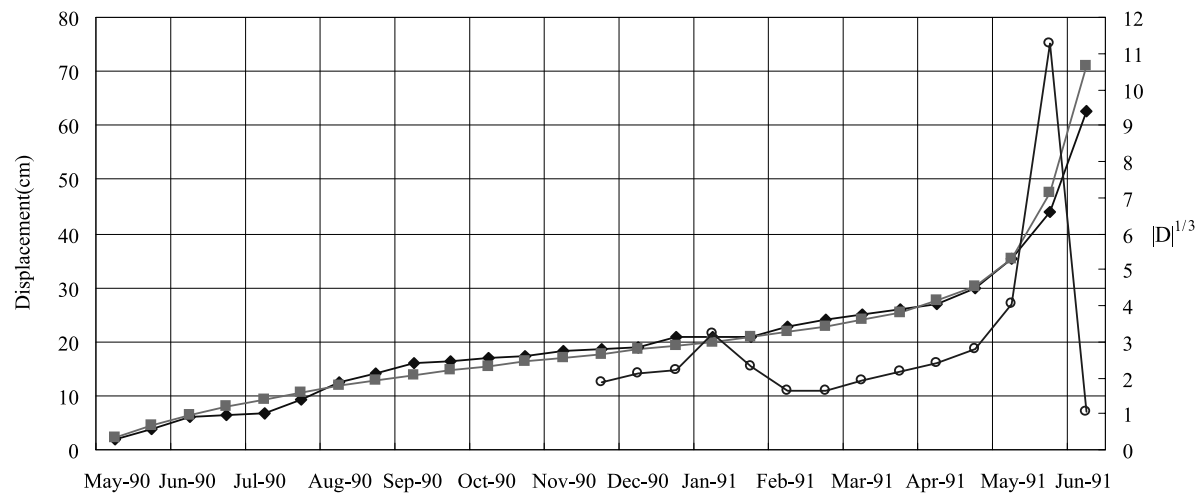
Time(months)

(b)

$$
\multimap-\text { Monitoring series } \quad-\text { Forecasting series } \quad-\mathrm{D}
$$

Fig. 9. Curves of the monitored values, forecasting ones and the catastrophic characteristic index $|D|$ versus time at C4 (a) and C7 (b) observation points

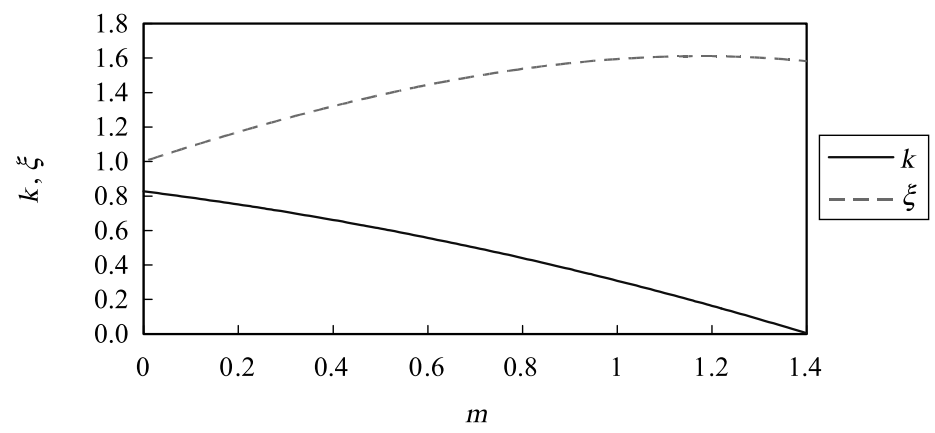

Fig. 10. Variations of $k$ and $\xi$ versus $m$ 


\section{Simplified Nonlinear Dynamic Model}

When the slope system evolves into the secondary creep phase, then $b=0$ and therefore Eq. (19) can be simplified as

$$
\frac{d x}{d t}=\left(\lambda_{1} x-\lambda_{2} x^{3}\right)=\lambda_{2}\left(\lambda x-x^{3}\right),
$$

where $\lambda_{1}=c a, \lambda_{2}=-c>0$ and $\lambda=\lambda_{1} / \lambda_{2}=-a$. Equation (27) represents a simplified nonlinear dynamic model. $\lambda_{1}<0(\lambda<0)$ and $\lambda_{1} \geq 0(\lambda \geq 0)$ denote that the slope is in the stable and unstable states, respectively.

\subsection{Bifurcation}

The parameter $\lambda$ in Eq. (27) is a control parameter. According to the Lyapunov's linear stability analysis theory (Wolf et al., 1985), it is easily shown that two groups of static solutions of Eq. (27) are as follows:

$$
\left\{\begin{array}{l}
\lambda<0, x_{0}=0, \omega=\lambda_{2} \lambda<0: x_{0} \text { is asymptotically stable } \\
\lambda>0,\left\{\begin{array}{l}
x_{0}=0, \omega=\lambda_{2} \lambda>0: x_{0} \text { is unstable } \\
x_{ \pm}= \pm \sqrt{\lambda}, \omega=-2 \lambda_{2} \lambda<0: x_{ \pm} \text {is asymptotically stable }
\end{array}\right.
\end{array}\right.
$$

where $\omega$ is the eigenvalue of Eq. (27). It is found from Eq. (28) that for the same static solution $x_{0}=0$, the system becomes unstable at $\lambda=0$ from the previously asymptotic stability, and hence produces two new asymptotically stable sub-solutions with an increment rate of $\sqrt{\lambda}$, when $\lambda$ changes from a negative value to a positive one. This shows that the system behaves as a bifurcation behavior and $\lambda=0(k=1)$ is the bifurcation point (Fig. 11). Also, this demonstrates that the conclusion drawn by the linear stability analysis is consistent with that by the catastrophe analysis, because $\lambda<0, \lambda=0$ and $\lambda>0$ correspond to $k>1, k=1$ and $k<1$, respectively.

\subsection{Chaos}

If the values of $x$ are measured at a fixed time interval $\Delta t$, then, the previously continuous variables $x(t)$ and $t$ become discrete ones $\left(x_{0}, x_{1}, x_{2}, \ldots\right)$, where $x_{\mathrm{n}}$ denotes

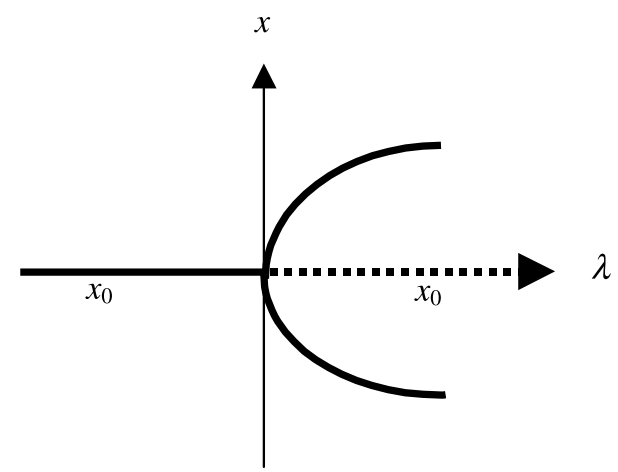

Fig. 11. The stability and bifurcation of the system (the thick real line and thick broken line denote asymptotic stability and unstability, respectively) 
the value $x$ measured at the nth time $(n=0,1,2, \ldots)$. Accordingly the continuous differential Eq. (27) becomes the following discrete difference Eq. (29), i.e.

$$
x_{n+1}=\left(1+\lambda_{2} \lambda\right) x_{n}\left(1-\frac{\lambda_{2}}{1+\lambda_{2} \lambda} x_{n}^{2}\right) \text {. }
$$

Let

$$
\begin{gathered}
\alpha=1+\lambda_{2} \lambda=1+c a \\
y_{n}=\left[\lambda_{2} /\left(1+\lambda_{2} \lambda\right)\right]^{1 / 2} x_{n}
\end{gathered}
$$

and Eq. (29) is rewritten as

$$
y_{n+1}=\alpha y_{n}\left(1-y_{n}{ }^{2}\right) .
$$

By applying an iterative computation for Eq. (32), it is found from Fig. 12 that the system has the solution with the period of 1 when $\alpha \leq 2$. As $\alpha$ increases, the solutions with the periods of $2,4,8$ and 16 can be obtained, respectively, corresponding to $\alpha=2.01,2.24,2.289$ and 2.299. When $\alpha>2.3$, there occurs a solution with the period of $2^{\infty}$. In other words, the period becomes infinite, i.e. the system does not hold

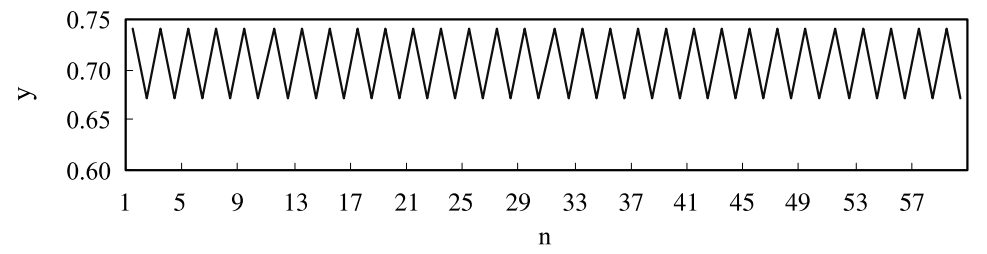

(a) Solutions with the period of 2 at $\alpha=2.01$

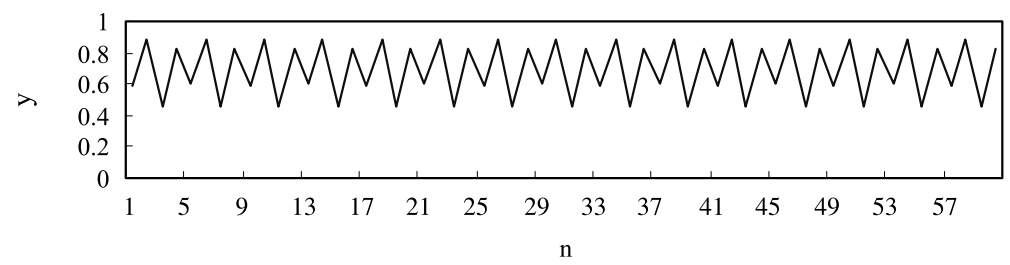

(b) Solutions with the period of 8 at $\alpha=2.289$

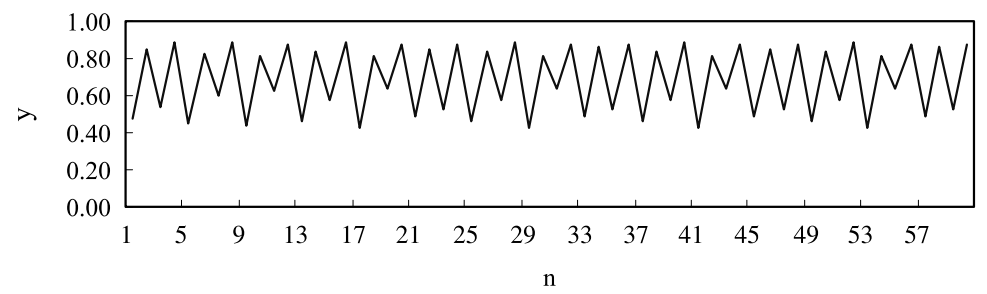

(c) Solutions with the period of $2^{\infty}$ at $\alpha=2.31$

Fig. 12. Curves of $y$ versus $n$ as $\alpha$ increases when solutions are stable 
periodicity any more and chaos appears. This analysis also shows that the evolution route leading to chaos of the slope system is obtained by the bifurcation of perioddoublings.

Substituting Eqs. (8) and (21) into Eq. (30) leads to

$$
\lambda_{1}=c a=\frac{G_{s}}{\eta}(1-k) m \exp \left(-\frac{m+1}{m}\right)>1.3
$$

We can judge whether the slope movement is chaotic or not by Eq. (33). It is noted that the unit of $\eta$ (generally, Pa.s) should be consistent with the time unit of $\Delta t$ while using Eq. (33). It is known from Eq. (33) that whether or not the chaotic phenomenon appears depends on the mechanical parameters $G_{\mathrm{s}}, \eta$ and $m$ of the strain-softening medium as well as the stiffness ratio $k$. Obviously, the larger $m$ is and the smaller $k$ is, the more prone to the occurrence of chaos the system is.

The physical implication of Eq. (33) is that when $a<0(k<1)$, i.e. a slope has the possibility of instability, the slope system may evolve into the chaotic state; whereas a slope is extraordinarily stable, no chaotic phenomenon appears. This means that chaos also is an indicator of the possibility of instability of the slope. The reasons can be explained as follows: for extremely stable slopes, the environmental factors, such as four-season climate, temperature, rainfall, earthquake and vibration, have little impact on their evolutionary states; contrarily, for possibly unstable slopes, especially those being in the critical state, the environmental factors have a great influence on their evolutionary state, which indicates that the evolution of these slopes is sensitive to the change of the environmental factors and therefore they could exhibit non-periodic motion (chaos). If a slope system has no chaotic characteristics, a deterministic prediction can be made; otherwise, the predictable time scale (Qin et al., 2001c) must be considered and the accuracy of prediction during the time scale should be studied.

\subsection{Simplified Physical Forecasting Model}

Substituting Eq. (7) into Eq. (27) in order to transform the variable $x$ into the variable $u$, one has

$$
\frac{d u}{d t}=\lambda_{1}\left(\frac{u-u_{1}}{u_{1}}\right)-\lambda_{2}\left(\frac{u-u_{1}}{u_{1}}\right)^{3} .
$$

Equation (34) is similar to the Logistic equation and is a simplified physical forecasting model.

Conducting the time prediction of landsliding in terms of the observed data of a landslide, one can solve the parameters $\lambda_{1}$ and $\lambda_{2}$ using the least squares method. The maximum displacement value which can be easily derived from Eq. (34) can be expressed as

$$
u_{\max }=u_{1}\left(1+\sqrt{\frac{\lambda_{1}}{3 \lambda_{2}}}\right) .
$$

The occurrence time of landsliding can be estimated according to the time corresponding to $\mathrm{u}_{\max }$. For the Jimingsi landslide, for example, we obtain $u_{\max }=72.2 \mathrm{~cm}$ and accordingly the unstable time is on 26 April 1991, using the observation series in the 
secondary creep phase at $\mathrm{C} 4$ observation point. The computed value of $\lambda_{1}$ is $0.171<1.3$, indicating no chaotic phenomenon of this landslide and that a deterministic prediction can be made.

\section{Conclusions}

This paper presents a cusp catastrophe model based on the catastrophe theory and discusses the necessary and sufficient conditions leading to landslide instability. It is assumed that the sliding surface of the landslide is planar and is a combination of two media: one is elastic-brittle or strain-hardening and the other is strain-softening. The shear stress-strain constitutive model for the strain-softening medium is described by the Weibull's distribution law. The conditions leading to a fast-moving landslide are derived. The following findings can be obtained from this paper:

1. The instability of a slope relies mainly on the ratio of the stiffness of medium 1 to the post-peak stiffness of medium 2, and the homogeneity index of medium 2 .

2. A newly-found role of water is to enhance the material homogeneity or brittleness and hence to reduce the stiffness ratio of the system.

3. A nonlinear dynamic model of the evolutionary process of a slope is presented by taking into account the viscosity or creep property of medium 2 . If the mechanical and geometric parameters for a slope are determined, a physical prediction can be made.

4. The parameters of the nonlinear dynamic model can be obtained from the observation data of a landslide by an algorithm of inversion. The observation series contains plenty of information associated with the mechanical parameters that can be estimated using the model and the algorithm presented in this paper.

5. The nonlinear dynamic analysis on the Jimingsi landslide shows that the catastrophic characteristic index $|D|$ has a relatively steady variation during the secondary creep phase. Then, this index shows a quick rise till reaching an extremely high peak value after entering into the tertiary creep, and in succession closes to zero prior to instability. We can judge the occurrence of landslide from this feature and regard the index $D$ as a parameter reflecting the precursory abnormality of a landslide.

6. A simplified nonlinear dynamic model is derived by considering the evolutionary characteristics of the slope being in the secondary creep. The properties of bifurcation and chaos of a landslide are studied. It is found that the bifurcation point, $k=1$, is the turning point of the slope system from stability to potential instability, and that chaos possibly arising from a potentially unstable slope can be generated in a manner of the bifurcation of period-doublings. The discriminant leading to chaos presented in this paper shows that the chaotic phenomenon is related to the mechanical parameters of the media along the sliding surface.

\section{Acknowledgements}

The work was funded by China national 973 programme (No. 2002CB412702). 


\section{References}

Bakus, G., Gilbert, F. (1970): Uniqueness in the inversion of inaccurate gross earth data. Philos. Trans. R. Soc. London Ser. A266(1173), 123-192.

Gehle, C., Kutter, H. K. (2003): Breakage and shear behaviour of intermittent rock joints. Int. J. Rock Mech. Min. Sci. 40, 687-700.

Henley, S. (1976): Catastrophe theory models in geology. Math. Geol. 8(6), 649-655.

Hudson, J. A., Fairhurst, C. (1969): Tensile strength, Weibull's theory and a general statistical approach to rock failure. In: Te'eni, M. (ed.), Proc. Southampton 1969 Civil Engineering Materials Conference (Part 2), 901-904.

Jaeger, J. C., Cook, N. G. W. (1979): Fundamentals of rock mechanics (third edition). Chapman and Hall, London.

Keilis-Borok, V. I. (1990): The lithosphere of the earth as a nonlinear system with implications for earthquake prediction. Rev. Geophys. 28, 19-34.

Lajtai, E. Z. (1967): The influence of interlocking rock discontinuities on compressive strength (model experiments). Felsmech. Ingenieurgeol. 5, 217-228.

Lu, G. F. (1994): Formation, monitoring and forecasting of Jimingsi landslide. Chin. J. Geol. Hazard Control 5[suppl.], 376-383.

Phillips, J. (1995): Nonlinear dynamics and the evolution of relief. Geomorphology 14, $57-64$.

Qin, S. Q., Zhang, Z. Y., Wang, S. T. (1993): An introduction to nonlinear engineering geology (in Chinese). Chinese Southwestern Traffic University Press, Chengdu.

Qin, S. Q., Wang, S. J. (2000): A homomorphic model for identifying abrupt abnormalities of landslide forerunners. Engng. Geol. 57, 163-168.

Qin, S. Q., Jiao, J. J., Wang, S. J. (2001a): A cusp catastrophe model of instability of slip-buckling slope. Rock Mech. Rock Engng. 34(2), 119-134.

Qin, S. Q., Jiao, J. J., Wang, S. J., Long, H. (2001b): A Nonlinear catastrophe model of instability of planar-slip slope and chaotic dynamical mechanisms of its evolutionary process. Int. J. Solids Struct. 38, 8093-8109.

Qin, S. Q., Wang, S. J., Jiao, J. J. (2001c): The predictable time scale of landslide, Bull. Engng. Geol. Environ. 59, 307-312.

Qin, S. Q., Jiao, J. J., Wang, S. J. (2002): A nonlinear dynamical model of landslide evolution. Geomorphology 43, 77-85.

Rat, M. (1988): Difficulties in foreseeing failure in landslides-La Clapière, French Alps. In: Proc., $5^{\text {th }}$ ISL, Lausanne 3, 1503-1505.

Saito, M. (1969): Forecasting time of slope failure by tertiary creep. In: Proc., Seventh Int. Conf. on Soil Mech. and Found. Engng., Montreal, 667-683.

Saunders, P. T. (1980): An introduction to catastrophe theory. Cambridge University Press, London.

Tang, C. A. (1997): Numerical simulation of progressive rock failure and associated seismicity. Int. J. Rock Mech. Min. Sci. 34(2), 249-261.

Tang, C. A. (1993). Catastrophe in rock unstable failure (in Chinese). China Coal Industry Publishing House, Beijing.

Tang, C. A., Hudson, J. A., Xu, X. (1993): Rock failure instability and related aspects of earthquake mechanisms (in Chinese). China Coal Industry Publishing House, Beijing. 
Thom, R. (1972): Stabilité structurelle et morphogénèse. Benjamin, New York.

Voight, B. (1989): Materials science law applies to time forecasts of slope failure. Landslide News (3), 8-11.

Wolf, A., Swift, J. B., Swinney, H. L., Vastano, J. A. (1985): Determing Lyapunov exponents from a time series. Physica 16D, 285-317.

Authors' address: Dr. Siqing Qin, Chinese Academy of Sciences, Engineering Geology and Applied Geophysics Department, 100029 Beijing, P.R. China; e-mail: qsqhope@ sohu.com 